\title{
A New Rotation Invariant Weber Local Descriptor for Recognition of Skin Diseases
}

\author{
Anabik Pal ${ }^{1}$, Nibaran Das ${ }^{2, *}$, Somenath Sarkar ${ }^{3}$, \\ Dwijendranath Gangopadhyay ${ }^{4}$, and Mita Nasipuri ${ }^{2}$ \\ ${ }^{1}$ Department of Information Technology \\ ${ }^{2}$ Department of Computer Science and Engineering \\ Jadavpur University, Kolkata (W.B), India \\ nibaran@gmail.com \\ ${ }^{3}$ Department of Dermatology, School of Tropical Medicine, Kolkata (W.B), India \\ ${ }^{4}$ Department of Dermatology, Burdwan Medical College, Burdwan (W.B), India
}

\begin{abstract}
A new rotation invariant Weber Local texture Descriptor(WLD) is proposed here. Performance of the developed features is evaluated on the basis of the recognition accuracies of SVM classifiers, trained and tested with the extracted features from the images of skins affected with three popular skin diseases such as Leprosy, Tineaversicolor, Vitiligo, and also normal skin, collected from School of Tropical Medicine, Kolkata. The WLD features are extracted with variations of the radius from 1 to 3 considering perimeters of having 8,16 and 24 pixels respectively. The modified WLD provides an average improvement of $4.79 \%$ in recognition accuracy over the normal WLD in the present four class problem. Dividing each sample image into 4 sub-regions through its centre of gravity and extracting WLD features from each of them. Thus we have extracted two different feature sets having normal WLD and rotation invariant WLD. They provide the maximum recognition accuracies of $85.06 \%$ and $87.36 \%$ respectively using SVM classiifiers on test set.
\end{abstract}

Keywords: Texture, WLD, SVM, Leprosy, Tineaversicolor, Vitiligo.

\section{Introduction}

Nowadays different types of texture features are popularly used in computer vision problems in many areas such as industrial automation, biomedical image processing, remote sensing, biometric identification, face recognition, robot vision etc. In general, texture representations are mainly of three types namely Statistical, Spectral, and Structural. For statistical approaches different statistical moments are used to describe the texture of digital images. These approaches are based on Gray Level Cooccurrence matrix [1,2], run length matrix[3], auto correlation, auto regression, MRF, moments etc. as found in literature. Spectral approach analyzes image texture in the frequency domain. As textures are periodic patterns, the entire frequency domain contains as much information as the image itself. Using this concept, several texture

\footnotetext{
* Corresponding author. 
descriptors have been proposed. For structural approaches the basic scheme is to build a grammar for the texture and then parse the texture to see if it matches the grammar. The idea can be extended by defining texture primitives, simple patterns from which more complicated ones can be built. In that case, the parse tree for the pattern in a particular region can be used as a descriptor. Recently some local descriptors such as Local Binary Pattern(LBP)[4], Local Directional Pattern (LDP)[5], Weber Local Descriptor (WLD)[6] have been proposed. Among which, LBP is very popular compared to others. This is due to its easy implementation, short execution time, gray scale and rotation invariant property. Though WLD is more robust than LBP for noisy images, but the original WLD feature set is not rotation invariant. This motivated us to introduce rotation invariant property into the WLD feature set. Here the developed feature set is used to recognize three popular skin diseases such as Leprosy, Tineaversicolor and Vitiligo whose manifestations are all but similar in color. Later sample images are divided into four sub-regions by partitioning through it's center of gravity (CG) from where WLD features are extracted. This process emphasizes the local information in the feature set.

\section{Weber Local Descriptor (WLD)}

Weber's Law[7] states that the ratio of the increment threshold to the background intensity is a constant. This can be represented as

$$
\frac{\Delta}{I}=k
$$

where $\Delta \mathrm{I}$ is the increment threshold, which signifies the noticeable difference in ' $\mathrm{I}$ ' for discrimination, ' $\mathrm{I}$ ' represents the initial stimulus intensity, and ' $\mathrm{k}$ ' is a constant. The fraction of the equation 1 is known as the Weber fraction. Using this law WLD has been proposed. It has two components 1) differential excitation ( $\xi$ ) and 2) orientation $(\theta)$. For computing WLD for any pixel, it's neighborhood has to be defined first. The neighborhood is generally circular with a fixed number of pixels on the perimeter of the circle. Thus, the neighborhood is defined in terms of the radius $\mathrm{R}$ of the circle and the number of neighboring pixels P. Some typical neighborhoods are shown in Figure 1.

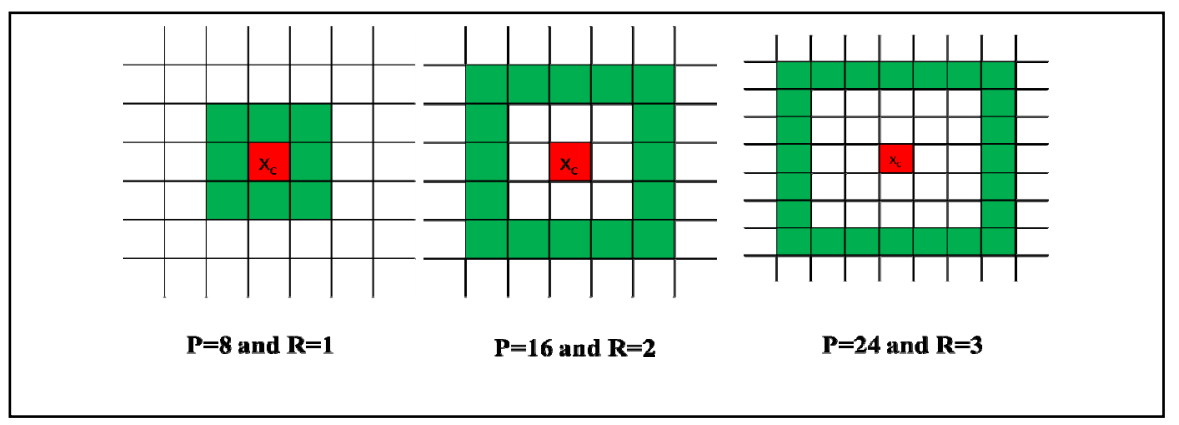

Fig. 1. Different neighborhood distribution 


\subsection{Differential Excitation $(\xi)$}

This component tries to find the salient variations within an image to simulate the pattern perception of human beings. To compute differential excitation $\xi\left(\mathrm{x}_{\mathrm{c}}\right)$ of a current pixel $\mathrm{x}_{\mathrm{c}}$, the sum of differences of intensities between it and its neighbors is calculated by using following equation .

$$
d_{I}^{0}=\sum_{i=0}^{P-1} \Delta I=\sum_{i=0}^{P-1}\left[I\left(\mathrm{x}_{i}\right)-I\left(\mathrm{x}_{c}\right)\right]
$$

where $x_{i}(i=0,1, \ldots \ldots, P-1)$ denotes the $i^{\text {th }}$ neighbors of $x_{c}$ and $P$ is the number of neighbors and $I\left(x_{i}\right)$ represents the intensity of the pixel $x_{i}$. Using Weber's Law, the ratio of the intensity differences to the intensity of the current pixel is then computed by using the following equation:

$$
k\left(\mathrm{x}_{c}\right)=\frac{d_{I}^{0}}{I\left(\mathrm{x}_{c}\right)}
$$

The differential excitation of the current pixel $\xi\left(\mathrm{x}_{\mathrm{c}}\right)$ is then computed as:

$$
\xi\left(x_{c}\right)=\arctan \left[k\left(x_{c}\right)\right]=\arctan \left[\sum_{i=0}^{p-1} \frac{I\left(x_{i}\right)-I\left(x_{c}\right)}{I\left(x_{c}\right)}\right]
$$

Obviously $\xi\left(\mathrm{x}_{\mathrm{c}}\right)$ takes negative value if the neighbors' intensities are smaller than that of the current pixel i.e. the surroundings are darker than the current pixel and takes positive values when the surroundings are lighter than the current pixel. To preserve this darker or brighter discriminating information $\xi\left(\mathrm{x}_{\mathrm{c}}\right)$ is not confined by taking absolute value of the numerator.

\subsection{Orientation}

The orientation component of WLD is computed as:

$$
\theta\left(x_{c}\right)=\arctan \left(\frac{d_{I}^{1}}{d_{I}^{2}}\right)
$$

where $d_{I}^{1}$ is the difference intensity between two diagonally opposite neighbors $d_{I}^{2}$ is the difference intensity between another pair of neighbors situated at the ends of a diagonal perpendicular to the previous one. For $\mathrm{P}=8$ and $\mathrm{R}=1$ the numerator and denominator are defined as below:

$$
\begin{aligned}
& d_{I}^{1}=I\left(x_{5}\right)-I\left(x_{1}\right) \\
& d_{I}^{2}=I\left(x_{7}\right)-I\left(x_{3}\right)
\end{aligned}
$$

\subsection{Rotation Invariant WLD Descriptor (WLDRI)}

The differential excitation is rotation invariant as it computes the sum of intensity difference of center pixel with neighbor pixels so we get same value for any orientation of image pixels. But orientation component of WLD will not be fixed if the image is rotated. So, to make orientation component rotation invariant, all possible mutually perpendicular diagonal pairs are considered for computing $\theta_{\mathrm{i}}$ as shown in equation 8 and out of them minimum one is chosen as shown in equation 9. 


$$
\begin{gathered}
\theta_{i}=\arctan \left(\frac{I\left(x_{((P / 2)+i) \bmod P}\right)-I\left(x_{i}\right)}{I\left(x_{((3 P / 4)+i) \bmod P}\right)-I\left(x_{((P / 4)+i) \bmod P}\right)}\right) \\
\theta\left(\mathrm{x}_{c}\right)=\min _{i=0}^{P-1}\left(\theta_{i}\right)
\end{gathered}
$$

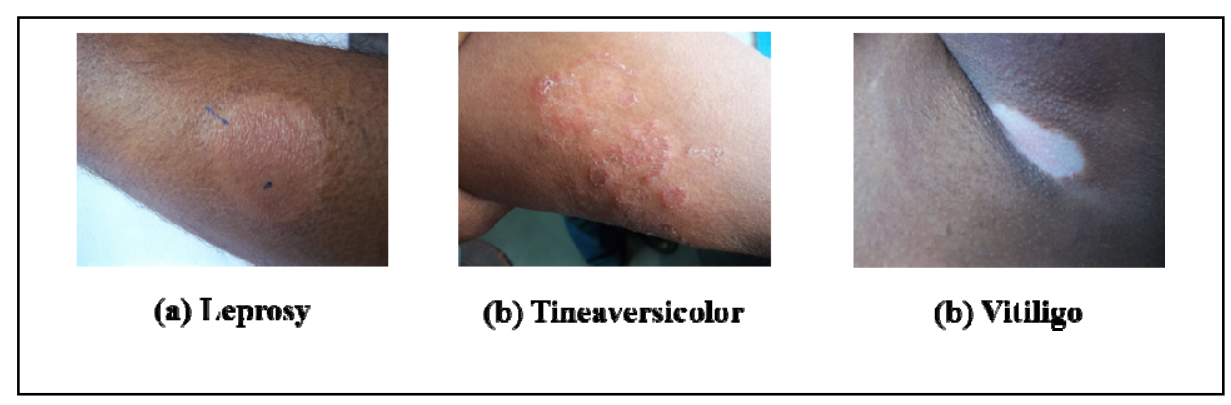

Fig. 2. Diseases affected skin images

\subsection{WLD Histogram}

Obviously both Differential Excitation and Orientation can take values in between $\pi / 2$ and $+\pi / 2$. The angle for Differential Excitation quantized into $\mathrm{T}$ equal width bins and for Orientation it is quantized into $M$ equal width bins. Now we get a two dimensional histogram $h_{t, m}$. This histogram gives the feature of the image. Two pixel get same $h_{t, m}$ value when these two pixels differential excitations belong to the same bin, and orientations are also belonging to the same bin.

\section{Experimental Setup}

As mentioned earlier, we have used the developed rotation invariant WLD for the recognition of three popular skin diseases named as Leprosy, Tineaversicolor and Vitiligo which are all but similar in color while affected. The color changes due to formation of hypo-pigmented skin lesions in Leprosy, Tineaversicolor and formation of de-pigmented skin lesions in Vitiligo. The color of the lesions becomes lighter than the normal skin color for all three cases. We have collected the images of the affected regions from the outdoor patients of department of Dermatology, School of Tropical Medicine (STM) during the period April 2011- March 2012. During that period, we have managed to get images of the affected skin regions from 141 patients whose diseases were properly diagnosed by the dermatologists of the STM. The KODAK Easy share 143 camera having 3X optical Zoom and 12 megapixel resolutions has been used for capturing those images. The patients, already under treatment, was not considered in our study; only new cases were included. Multiple images were captured from the patients having more than one skin lesions. It is worthy to mention here that the images of normal skin of patients were also captured during the procedure. We have selected 876 images randomly from the collected images. The images are divided into two parts training and testing set maintaining nearly 4:1 ratio for each 
of the four classes including normal skin. Some sample images of the affected regions are shown in Figure 2. The skin images are classified using a SVM based classification technique on the basis of WLD features (both WLD and WLDRI) extracted from those images. For WLD features, bins considers for $\xi\left(\mathrm{x}_{\mathrm{c}}\right)$ and $\theta\left(\mathrm{x}_{\mathrm{c}}\right)$ are 8 and 6 respectively. Here two variations in feature extraction process are considered as described below:

1) WLD features with fixed set of $(P, R)$ values are extracted from the whole image.

2) Each image sample is partitioned into four regions by drawing a horizontal and a vertical line through the $\mathrm{CG}$ of the image. Here partitioning is done for giving emphasis on regional features extracted from the image samples and CG is chosen as the partitioning point for getting similar gray level distribution in all the four regions. Let the $\mathrm{CG}$ is the co-ordinate $\mathrm{C}\left(\mathrm{c}_{\mathrm{x}}, \mathrm{c}_{\mathrm{y}}\right)$ then the equation for calculating $\mathrm{CG}$ of an image is given as below:

$$
C\left(c_{x}, c_{y}\right)=\left(\frac{\sum_{i=0}^{M} \sum_{j=0}^{N} \mathrm{i} . f(\mathrm{i}, \mathrm{j})}{\sum_{i=0}^{M} \sum_{j=0}^{N} f(\mathrm{i}, \mathrm{j})}, \frac{\sum_{i=0}^{M}}{\sum_{i=0}^{M} \sum_{j=0}^{N} \mathrm{j} . f(\mathrm{i}, \mathrm{j})}\right)
$$

Here the dimension of the image is $M \times N$ and $f(i, j)$ is the intensity value at $(i, j)$. WLD features with fixed set of $(P, R)$ values are extracted from each of the four sub regions which are concatenated to form the feature set variations 1 and 2, mentioned above are repeated with different sets of values for P and R. Finally the features extracted in variation 3 are concatenated to form a new set.

\section{$4 \quad$ Result and Discussion}

The above mentioned features extraction techniques are applied to obtain different feature sets. The different sets of $\mathrm{P}$ and $\mathrm{R}$ values considered here are $\mathrm{P}=8$ and $\mathrm{R}=1$, $\mathrm{P}=16$ and $\mathrm{R}=2, \mathrm{P}=24$ and $\mathrm{R}=3$. The classification ability of these feature sets is tested by using multiclass SVM available in Weka 3.7.9 [8] tool. Train dataset is constructed here with 702 image samples and the rest 174 image samples are used as a test dataset for the present work. The recognition accuracy achieved with each feature extraction process is listed in Table1.We see that in all cases WLDRI is performed better than normal WLD and CG based partitioning increases the accuracy.

Table 1. Recognition accuracies obtained from different feature extraction techniques

\begin{tabular}{|c|l|c|c|}
\hline \multirow{2}{*}{ No } & \multirow{2}{*}{ Feature set used } & \multicolumn{2}{c|}{ Recognition Accuracy (in \%) } \\
\cline { 3 - 4 } & & Without Partition & CG Based Partition \\
\hline 1. & WLD T=8,M=6,P=8,R=1 & 72.98 & 74.14 \\
\hline 2. & WLD T=8,M=6,P=16,R=2 & 74.71 & 78.16 \\
\hline 3. & WLD T=8,M=6,P=24,R=3 & 71.84 & 85.06 \\
\hline 4. & WLDRI T=8,M=6,P=8,R=1 & 74.71 & 78.74 \\
\hline 5. & WLDRI T=8,M=6,P=16,R=2 & 79.31 & 82.76 \\
\hline 6. & WLDRI T=8,M=6,P=24, $\mathrm{R}=3$ & 79.88 & 87.36 \\
\hline 7. & Concatenation of feature set 1,2 and 3 & 83.91 & 86.78 \\
\hline 8. & Concatenation of feature set 4,5 and 6 & 85.06 & $\mathbf{8 9 . 0 8}$ \\
\hline
\end{tabular}




\section{Conclusion}

In the present work, a new rotation invariant Weber Local texture descriptor (WLD) is proposed to identify three similar looking skin diseases such as Leprosy, Tineaversicolor and Vitiligo on the basis of the performance of SVM classifier. The developed feature performs better than the original WLD and provides an average improvement of $4.79 \%$ in recognition accuracy in the present four class problem. We have also proposed a CG based region partitioning scheme to divide sample images into 4 subregions for extraction of more local information from every sub-regions. This provides the maximum recognition accuracy of $85.06 \%$ and $87.36 \%$ for normalfor normal WLD and rotation invariant WLD respectively. Multiscale analysis of both the features are also done here by choosing three sets of $(\mathrm{P}, \mathrm{R})$ values and a highest recognition accuracy of $89.08 \%$ is achieved with WLDRI and CG based partitioning. The obtained recognition accuracy is quite satisfactory. Introduction of stronger feature set and/or with different classifier combination or fusion techniques may be explored further.

Acknowledgment. Authors are grateful to the "Center for Microprocessor Application for Training Education and Research" of Computer Science \& Engineering Department, Jadavpur University laboratory, for providing infrastructure facilities during progress of the work. Authors are also thankful to Department of dermatology, School of Tropical medicine, Kolkata for providing patients and medical expertise during data collection drive.

\section{References}

[1] Haralick, R.M., et al.: Textural Features for Image Classification. IEEE Transactions on Systems, Man and Cybernetics SMC-3, 610-621 (1973)

[2] Haralick, R.M.: Statistical and structural approaches to texture. Proceedings of the IEEE 67, 786-804 (1979)

[3] Galloway, M.M.: Texture analysis using gray level run lengths. Computer Graphics and Image Processing 4, 172-179 (1975)

[4] Ojala, T., et al.: Multiresolution gray-scale and rotation invariant texture classification with local binary patterns. IEEE Transactions on Pattern Analysis and Machine Intelligence 24, 971-987 (2002)

[5] Jabid, T., et al.: Gender Classification using Local Directional Pattern (LDP). Presented at the International Conference on Pattern Recognition (2010)

[6] Jie, C., et al.: WLD: A Robust Local Image Descriptor. IEEE Transactions on Pattern Analysis and Machine Intelligence 32, 1705-1720 (2010)

[7] Jain, A.K.: Fundamentals of Digital Signal Processing: Prentice-Hall (1989)

[8] Hall, M., et al.: The WEKA data mining software: an update. ACM SIGKDD Explorations Newsletter 11, 10-18 (2009) 